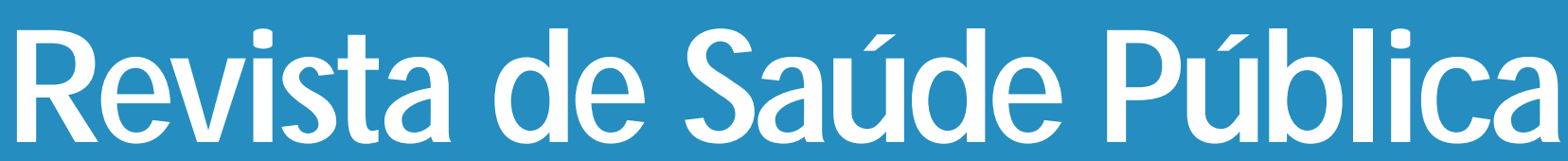

$\begin{array}{llllllll}\mathbf{J} & \mathbf{O} & \mathbf{U} & \mathbf{R} & \mathbf{N} & \mathbf{A} & \mathbf{L}\end{array}$

$0 F$

$\mathbf{P} \mathbf{U}$

B L I C

H E A L T H

\title{
Infecção pelo HIV: descritores de mortalidade em pacientes hospitalizados*
}

\section{Mortality descriptors in HIV inpatients}

Luiz Claudio Santos Thuler, André Luís Hatherly, Patrícia Nascimento G óes e Jorge Roberto de Almeida e Silva

Hospital Universitário Gaffrée e Guinle da Escola de Medicina e Cirurgia da Universidade do Rio de Janeiro. Rio de Janeiro, RJ - Brasil mortalidade em pacientes hospitalizados* Rev. Saúde Pública, 32 (6): 572-8, 1998

(c) Copyright Faculdade de Saúde Pública da USP. Proibida a reprodução mesmo que parcial sem a devida autorização do Editor Cient́fico. Proibida a utilização de matérias para fins comerciais. All rights reserved. 


\title{
Infecção pelo HIV: descritores de mortalidade em pacientes hospitalizados*
}

\section{Mortality descriptors in HIV inpatients}

\author{
Luiz Claudio Santos Thuler, André Luís Hatherly, Patrícia N ascimento G óes \\ e Jorge Roberto de Almeida e Silva \\ Hospital Universitário Gaffrée e Guinle da Escola de Medicina e Cirurgia da Universidade do Rio de \\ Janeiro. Rio de Janeiro, RJ - Brasil
}
Objetivo Estudar os descritores clínico-epidemiológicos da mortalidade em pacientes internados por condições clínicas associadas à infecção pelo HIV.
Método Estudo retrospectivo de todos os pacientes adultos hospitalizados em 1990, 1992 e 1994 em hospital universitário. Os resultados foram descritos como números absolutos, percentagens e médias, sendo a significância estatística entre as diferenças avaliada pelos testes do qui-quadrado, exato de Fisher ou t de Student, conforme o caso. Um modelo de regressão logística foi elabora- do visando a identificar os principais fatores associados ao risco de evolução para o óbito.

Resultados Foram incluídos no estudo 240 pacientes. Entre 1990 e 1994 a idade média dos pacientes aumentou de 35,0 para 36,9 anos, a razão entre os sexos masculino e feminino caiu de 9,8 para 2,0, a proporção de não brancos cresceu de 18,5 para 41,3 e registrou-se um aumento do tempo médio entre a descoberta da infecção pelo HIV e a hospitalização de 0,7 para 2,5 anos. Observou-se a redução do número médio de dias de hospitalização de 31,3 para 25,3 e aumento da proporção de pacientes em acompanhamento ambulatorial de 47,8 para $83,3 \%$. As infecções respiratórias representaram a principal causa de hospitalização $(58 \%)$ e as infecções oportunistas apresentadas com maior freqüência foram: candidíase oral $(27,1 \%)$, tuberculose $(18,3 \%)$, pneumonia por Pneumocystis carinii $(15,4 \%)$ e neurotoxoplasmose $(10,4 \%)$. Na análise multivariada, apenas o tempo de hospitalização menor ou igual a 7 dias (Odds Ratio $[\mathrm{OR}]=3,88$; $\mathrm{p}=0,02)$ e a ausência de acompanhamento ambulatorial $(\mathrm{OR}=3,29 ; \mathrm{p}=0,01)$ mostraram-se associados a um maior risco de evolução para óbito.

Conclusão $\mathrm{O}$ conhecimento dos fatores associados a um risco aumentado de morte pode ser útil na tomada de decisão frente a pacientes hospitalizados com infecção pelo HIV.

Síndrome de imunodeficiência adquirida. Tempo de intemação. Mortalidade hospitalar.

\footnotetext{
*Trabalho realizado com bolsa do Programa de Extensão da Universidade do Rio de Janeiro (UNI-RIO), 1994/1995.

Correspondência para/Correspondence to: Luiz Claudio Santos Thuler - Rua Visconde de Pirajá, 500/503 - Ipanema - 22410-003 Rio de Janeiro, RJ Brasil. E-mail: 1thuler@spacenet.com.br.

Recebido em 15.12.1997. Aprovado em 25.5.1998.
} 


\begin{abstract}
Objective To assess the clinical-epidemiological descriptors of inpatient mortality in persons infected with the human immunodeficiency virus (HIV).

Method All adult HIV/AIDS patients hospitalized at a university hospital in 1990, 1992 and 1994 were studied retrospectively. Descriptive statistics for all variables of interest were generated. Chi-square test and Fisher's exact test were performed to compare categorical variables. Means were compared using the Student $\boldsymbol{t}$ test. Stepwise logistic regression was used to identify the odds of dying associated with each risk factor.

Results Two hundred and forty patients were included in the study. Between 1990 and 1994 the mean age rose from 35 to 36.9 years, the male/female ratio decreased from 9.8 to 2.0, the non-white proportion increased from 18.5 to $41.3 \%$ and the mean time between detection of HIV infection and hospitalization rose from 0.7 to 2.5 years. In addition, length of stay dropped from 31.3 to 25.3 days and the proportion of patients being followed up in the outpatient clinic of the Universitary Hospital increased from 47.8 to $83.3 \%$. Respiratory infections were the main cause of hospitalization (58.0\%). Oral candidiasis $(27.1 \%)$, tuberculosis (18.3\%), Pneumocystis carinii pneumonia (15.4\%) and toxoplasmic encephalitis (10.4\%) were the most frequent opportunistic infections. Multivariate analysis showed that the factors associated with a worse outcome included the length of stay less than or equal to 7 days (Odds Ratio [OR]=3.88; $p=0.02)$ and no outpatient follow-up at the Hospital $(O R=3.29 ; p=0.01)$.
\end{abstract}

Conclusion Identification of independent risk factors for death may help in the implementation of more efficient interventions directed towards inpatients with HIV/AIDS.

Acquired immunodeficiency syndrome. Lenth of stay. Hospital mortality.

\section{INTRO DUÇÃO}

Descrita inicialmente, em 1981, por Gottlieb e col. ${ }^{7}$, em cinco homossexuais do sexo masculino com pneumonia por Pneumocystis carinü, a Síndrome da Imunodeficiência Adquirida (AIDS) vem se mostrando uma importante causa de morbidade e mortalidade para os mais diferentes estratos populacionais, sendo incertas, até o momento, as possibilidades de controle dessa pandemia. Projeções da Organização Mundial da Saúde, divulgadas em novembro de 1997, estimavam que havia cerca de 30,6 milhões de pessoas vivendo com infecção ou doença causadas pelo vírus da imunodeficiência humana (HIV), sendo mais de $90 \%$ dessas em países em desenvolvimento. O número acumulativo de casos de AIDS, nessa época, foi estimado em 12,9 milhões, com 11,7 milhões de óbitos ${ }^{17}$, embora a Coalisão Global estime que o número de infectados possa chegar a 110 milhões no ano $2000^{13}$.

No Brasil, acredita-se que o primeiro caso de AIDS tenha ocorrido em 1980. Desde então tem-se observado mudanças radicais no seu comportamen- to. Hoje a Síndrome já foi detectada em todas as unidades federadas do País, e não se limita a grupos ou pacientes com comportamentos "diferenciados", inicialmente estigmatizados como "grupos de risco", mas representa um risco real para toda a população ${ }^{15}$. Até 28/2/98, foram notificados ao Ministério da Saúde 328.821 casos de AIDS, estimando-se que o número de infectados pelo HIV seja de cerca de 700.000. Destes casos conhecidos, 51,7\% evoluíram para o óbito.

A evolução dos conhecimentos e da utilização de recursos diagnósticos e terapêuticos fez com que a AIDS tivesse a sua história natural alterada. Com um comportamento agudo e rapidamente fatal observado nos primeiros casos, vem assumindo características de doença crônica, apresentando-se hoje, na maioria das ocasiões, como uma infecção com um longo período assintomático, que pode ser interrompido por infecções oportunistas ou neoplasias, com uma sobrevida que pode atingir mais de $10 \operatorname{anos}^{9,11}$. Na medida em que a infecção pelo HIV torna-se mais prevalente e que os avanços tecnológicos permitem aumentar a sobrevida dos pacientes infectados, cres- 
ce a demanda à rede de atenção médica para o diagnóstico, acompanhamento, tratamento e profilaxia da infecção e suas conseqüências. Dessa forma, há necessidade de que os cuidados de saúde sejam efetuados por toda a rede de serviços de saúde e não somente por instituições especializadas ou hospitais de referência.

Fleishman e col. ${ }^{6}$ mostraram que pacientes com AIDS, atendidos em dez grandes cidades norte-americanas, entrevistados entre março de 1991 e setembro de 1992, tiveram, em média, duas hospitalizações por ano. Os principais fatores associados à hospitalização foram os seguintes: nível educacional baixo, uso de drogas ilícitas endovenosas, raça negra e ausência de seguro saúde. Esses pacientes permaneceram internados, em média, de 9,5 a 13,7 dias, conforme se tratasse de hospitalização por infecção ou doença causadas pelo HIV, respectivamente. Em outro trabalho, Mor e col. ${ }^{12}$ encontraram que as admissões hospitalares foram mais comuns nos pacientes sintomáticos, naqueles com déficit da capacidade funcional, com infecção definidora de AIDS, com história de uso de drogas ilícitas, não-brancos e desempregados. Outros estudos norte-americanos têm mostrado que mulheres com AIDS são menos frequientemente hospitalizadas ${ }^{8}$ e apresentam maior risco de morrer ${ }^{10}$ que pacientes do sexo masculino. Os investigadores sugerem que fatores socioeconômicos como a pobreza, a falta de moradia, a violência doméstica e o uso de drogas ilícitas sejam responsáveis por tais achados. Turner e Ball ${ }^{16}$, analisando uma amostra de 258 hospitais americanos, encontraram um coeficiente de letalidade global de $17,3 \%$.Todavia, afirmam que pouco se conhece sobre o risco de morte para os pacientes hospitalizados por AIDS.

No Rio de Janeiro, Rapparini e col. ${ }^{14}$ estudando pacientes internados no Hospital Universitário Clementino Fraga Filho, da Universidade Federal do Rio de Janeiro (UFRJ), mostraram que, em 1988, $58,3 \%$ dos pacientes hospitalizados por condições clínicas relacionadas à AIDS evoluíam para o óbito durante a internação, enquanto que em 1994 esse coeficiente foi reduzido para $24,7 \%$.

Interessados em conhecer o perfil dos pacientes hospitalizados em um outro hospital universitário foi elaborado o presente estudo, que visa a identificar os principais descritores clínico-epidemiológicos da mortalidade hospitalar os em pacientes internados para o diagnóstico e tratamento de condições clínicas relacionadas à infeção pelo HIV.

\section{MÉTODO}

O hospital universitário estudado é um hospital terciário, com 206 leitos distribuídos entre as clínicas médicas e cirúrgicas. Predomina a atenção às doenças crônico-degenerativas e, mais recentemente, o hospital vem se destacando na área metropolitana do Rio de Janeiro como um dos centros de referência para o diagnóstico e tratamento de pacientes com a síndrome da imunodeficiência adquirida. Três serviços clínicos têm se ocupado da atenção aos pacientes com infecção pelo HIV: $10^{\mathrm{a}}$ enfermaria, $8^{\mathrm{a}}$ enfermaria e enfermaria de doenças infecciosas e parasitárias (DIP), sendo oferecido acompanhamento ambulatorial, hospitalização, apoio psicológico, avaliação nutricional, assistência social e testagem anônima, entre outros.

Foi realizado estudo retrospectivo de todos os pacientes adultos em primeira internação por condições clínicas relacionadas à infecção pelo HIV, em três diferentes períodos de 12 meses cada: 1 de janeiro a 31 de dezembro de 1990, 1 de janeiro a 31 de dezembro de 1992 e 1 de janeiro a 31 de dezembro de 1994. Foram eliminadas as reinternações. Os anos de 1991 e 1993 não foram considerados no estudo, por tratar-se de períodos atípicos em que ocorreram, respectivamente, greve de funcionários públicos e contenção de despesas hospitalares.

As populações foram tratadas de maneira independente. Foram analisadas as seguintes variáveis: idade, sexo, estado civil, comportamento de risco, infecções oportunistas e neoplasias diagnosticadas durante a hospitalização, tempo entre o diagnóstico da infecção pelo HIV e a internação, classificação clínica (critérios do Ministério da $\operatorname{Saúde}^{2}$ ) no momento da hospitalização, motivo da internação, número de dias de hospitalização, história de internação prévia em outra instituição, história de acompanhamento ambulatorial e evolução clínica .

Os dados colhidos foram analisados com o apoio dos programas Epi Info (versão 6.03, 1995) e LOGISTIC ${ }^{5}$ (versão 3.11Ef, 1994). Variáveis dicotômicas foram descritas em números absolutos e percentagens, sendo a significância estatística da diferença entre duas percentagens verificada pelos testes do qui-quadrado ou exato de Fisher, quando indicado. Para as variáveis contínuas foram apresentadas a média e o intervalo com $95 \%$ de confiança (IC 95\%), sendo a diferença estatística entre duas médias avaliada pelo teste $\mathbf{t}$ de Student. Por tratar-se de pequenos subgrupos a cada ano, para a identificação dos fatores independentemente associados ao óbito foi realizada análise multivariada considerando-se a população como um todo. O modelo de regressão logística contemplou as seguintes variáveis: hospitalização $\leq 7$ dias, não acompanhamento ambulatorial, idade $>40$ anos, não uso de droga anti-retroviral, sexo masculino, cor não-branca, infecção pelo HIV < 12 meses e internação anterior. Os riscos foram estimados como Odds Ratio (OR) e apresentados com os seus respectivos intervalos com $95 \%$ de confiança. 


\section{RESU LTADOS}

A análise dos dados referentes a 240 pacientes hospitalizados nos anos de 1990, 1992 e 1994 mostram que a idade variou entre 17 e 68 anos, com uma média de 36,3 anos (IC95\%= 17,7 - 54,9), sendo $77,9 \%$ do sexo masculino, $64,5 \%$ de cor branca e $75,0 \%$ solteiros. As informações contidas na Tabela I descrevem as variações, ao longo dos anos, de variáveis selecionadas, observando-se discreto aumento na média de idade dos pacientes (35 anos para 36,9, $\mathrm{p}=0,40$ ), redução significativa na razão entre o sexo masculino e o feminino $(9,8$ para 2,$0 ; \mathrm{p}=0,002)$ e aumento significativo no percentual de pacientes nãobrancos $(18,5 \%$ para $41,3 \%$; $\mathrm{p}=0,01)$. A distribuição percentual dos pacientes conforme o estado civil manteve-se estável no período estudado. Pode-se ainda constatar que o tempo médio entre a descoberta da infecção pelo HIV e a hospitalização foi de 1,8 anos, com uma tendência de aumento (de 0,7 para 2,5 anos; $p=0,00001$ ) ao longo dos anos. O mesmo se observa em relação ao percentual de hospitalizações de pacientes que já haviam apresentado previamente uma ou mais condições clínicas definidoras de AIDS conforme os critérios ${ }^{2}$ do $\mathrm{Mi}$ nistério da Saúde (43,1\% para 76,7\%; p=0,000005).

A Tabela 1 mostra que as complicações sobre o sistema respiratório podem ser implicadas como prin-

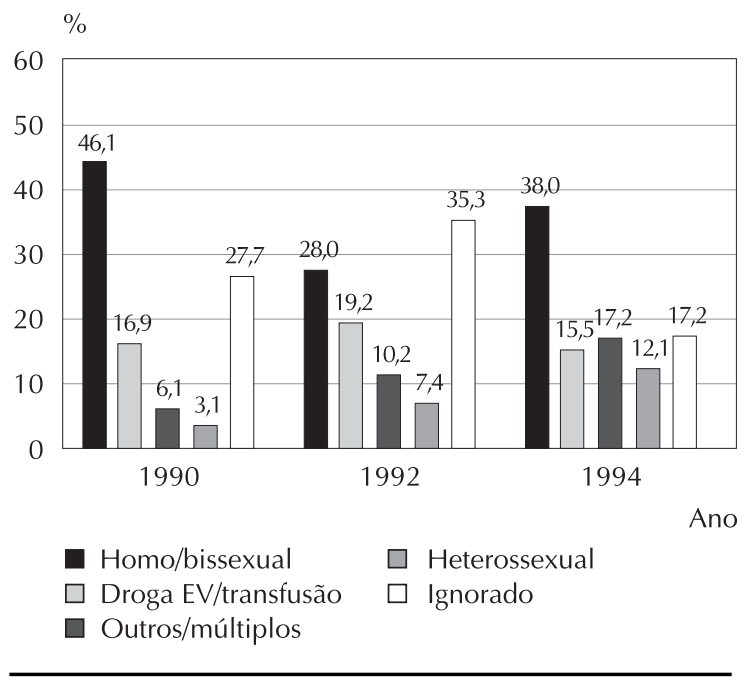

Figura 1 - Freqüência dos comportamentos de risco para a infecção pelo HIV conforme 0 ano de hospitalização. Hospital U niversitário Gaffrée e Guinle, 1990, 1992 e 1994.

Tabela 1 - Características clínico-epidemiológicas registradas no momento da hospitalização, conforme 0 ano, em 240 pessoas infectadas pelo HIV.

\begin{tabular}{|c|c|c|c|}
\hline \multirow{2}{*}{ Variável } & \multicolumn{3}{|c|}{ Ano } \\
\hline & 1990 & 1992 & 1994 \\
\hline $\mathrm{N}$ úmero de pacientes novos & 65 & 85 & 90 \\
\hline Idade, média & 35,0 & 36,7 & 36,9 \\
\hline \multicolumn{4}{|l|}{ Sexo, \%* } \\
\hline M asculino & 90,8 & 80,0 & 67,4 \\
\hline Feminino & 9,2 & 20,0 & 32,6 \\
\hline Razão masculino: feminino & 9,8 & 41,0 & 2,0 \\
\hline \multicolumn{4}{|l|}{ Cor, \%* } \\
\hline Branca & 81,5 & 69,6 & 58,8 \\
\hline O utra & 18,5 & 30,4 & 41,3 \\
\hline \multicolumn{4}{|l|}{ Estado civil, \% } \\
\hline Solteiro & 72,3 & 75,2 & 76,6 \\
\hline Casado & 23,1 & 12,9 & 15,5 \\
\hline Outro & 4,6 & 11,7 & 7,7 \\
\hline Anos entre a infecção pelo HIV e a hospitalização, média* & 0,7 & 2,2 & 2,5 \\
\hline \multicolumn{4}{|l|}{ Classificação clínica no momento da hospitalização, \% } \\
\hline Infecção pelo HIV & 56,9 & 23,5 & 23,3 \\
\hline AIDS & 43,1 & 76,5 & 76,7 \\
\hline \multicolumn{4}{|l|}{ M otivo da Internação, \% } \\
\hline Doença respiratória & 58,5 & 66,7 & 52,9 \\
\hline Doença diarréica & 33,8 & 46,4 & 44,7 \\
\hline Doença neurológica & 36,9 & 50,0 & 43,4 \\
\hline Dias de hospitalização, média & 31,3 & 20,4 & 25,3 \\
\hline História de internação prévia em outra instituição, \%* & 39,1 & 18,5 & 30,2 \\
\hline História de acompanhamento ambulatorial no HUGG, \%* & 47,8 & 65,6 & 83,3 \\
\hline Evolução para o óbito, \% & 36,9 & 45,6 & 51,2 \\
\hline
\end{tabular}


cipal causa de hospitalização nos três períodos cobertos pelo estudo. Observa-se ainda uma redução no valor médio de dias de hospitalização entre os anos de 1990 e 1992 e um pequeno aumento entre 1992 e 1994 ( p=0,06). Uma história de hospitalização anterior em outra instituição foi registrada em $28,6 \%$ dos casos e $60,4 \%$ dos pacientes vinham sendo acompanhados ambulatorialmente. Há um aumento progressivo no percentual de pacientes em acompanhamento ambulatorial nos anos do estudo (de 47,8 para $83,3 \% ; \mathrm{p}=0,02)$. Quarenta e cinco por cento dos pacientes evoluíram para óbito durante a hospitalização. Esse percentual mostra um aumento discreto ao longo dos anos, embora não seja estatisticamente significativo $(36,9$ para $51,2 \% ; \mathrm{p}=0,21)$.

Os prováveis modos de transmissão da infecção são apresentados na Figura 1. O comportamento homossexual/bissexual masculino pode ser implicado em $37,1 \%$ das vezes, seguindo-se do uso de drogas endo-venosas $(9,4 \%)$ e das transfusões de sangue e hemoderivados $(7,9 \%)$. Ressalta-se a existência de grande número de modos de transmissão ignorados $(27,2 \%)$, o que provavelmente reflete um sub-registro da informação.

O número médio de infecções oportunistas ou neoplasias foi de 1, 2 por internação, variando entre 0 e 7 . O percentual de pacientes acometidos por cada uma das principais condições clínicas definidoras de AIDS é apresentado na Figura 2, mostrando-se que $27,1 \%$ dos pacientes apresentaram candidíase oral, $18,3 \%$ tuberculose pulmonar e/ou extra-pulmonar e 15,4\% pneumonia por Pneumocystis carinii.

Uma análise multivariada de fatores selecionados (Tabela 2) mostrou que estiveram estatisticamente associados ao risco de evolução para o óbito as hospitalizações menores que 7 dias $(p=0,02)$ e a ausência de acompanhamento ambulatorial $(\mathrm{p}=0,01)$. Cor, idade e sexo do paciente, bem como história de internação anterior em outra instituição, infecção pelo HIV há menos de 12 meses e o não-uso de droga anti-retroviral foram excluídas do modelo por não apresentarem significância estatística.

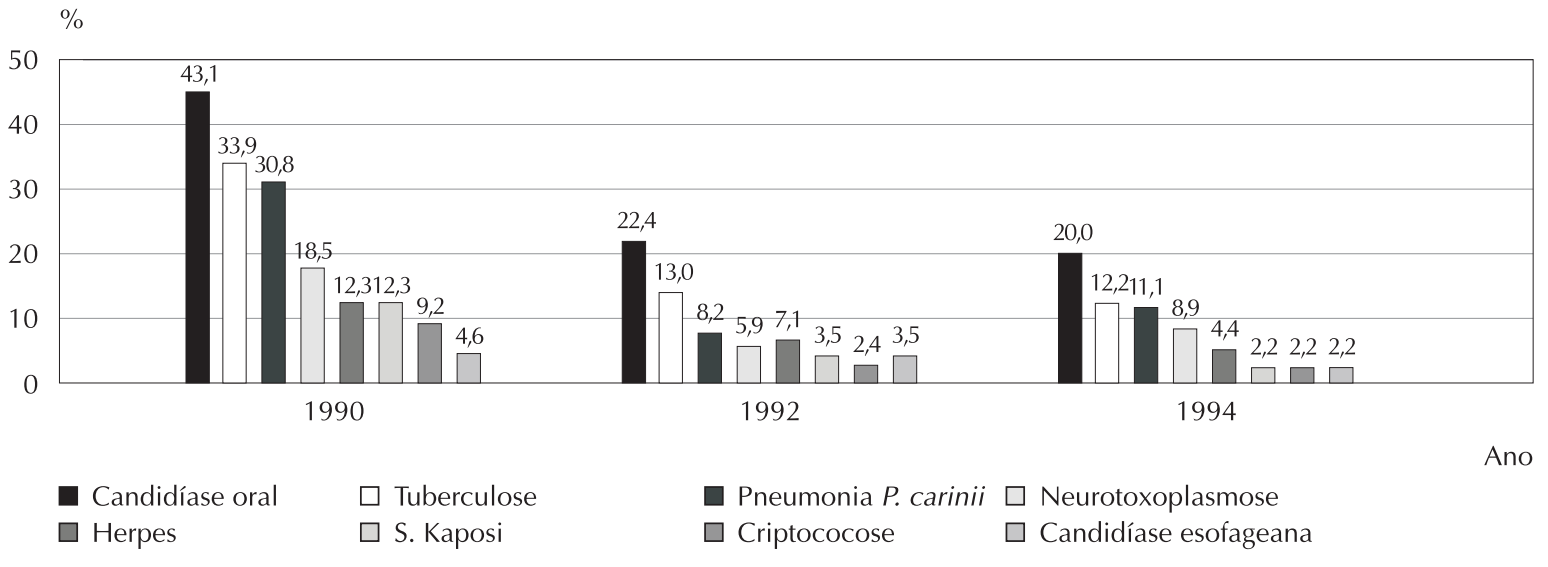

Figura 2 - Freqüência das principais infecções oportunistas e neoplasias diagnosticadas durante a hospitalização conforme o ano. Hospital Universitário Gaffrée e Guinle, 1990, 1992 e 1994.

Tabela 2 - Análise multivariada do risco de evolução para o óbito conforme a presença de variáveis selecionadas em 240 pessoas infectadas pelo HIV.

\begin{tabular}{llllll}
\hline Variável & $\begin{array}{c}\text { Altas } \\
(\mathrm{n}=136)\end{array}$ & $\begin{array}{c}\text { Ó bitos } \\
(\mathrm{n}=104)\end{array}$ & OR & $\begin{array}{c}\text { IC 95\% } \\
\text { para O R }\end{array}$ & $\begin{array}{c}\text { valor } \\
\text { de } \mathrm{p}\end{array}$ \\
\hline Hospitalização $\leq 7$ dias & $16,7 \%$ & $37,5 \%$ & 3,88 & $1,19-12,55$ & $\mathbf{0 , 0 2}$ \\
Não acompanhamento ambulatorial & $31,7 \%$ & $56,3 \%$ & 3,29 & $1,17-9,25$ & $\mathbf{0 , 0 1}$ \\
Idade > 40 anos & $21,4 \%$ & $33,7 \%$ & 2,04 & $0,67-6,21$ & 0,18 \\
Não uso de anti-retroviral & $87,8 \%$ & $94,8 \%$ & 1,66 & $0,28-9,89$ & 0,63 \\
Sexo masculino & $77,8 \%$ & $81,7 \%$ & 1,53 & $0,34-6,77$ & 0,41 \\
Cor não branca & $32,5 \%$ & $28,9 \%$ & 1,38 & $0,45-4,16$ & 0,90 \\
Infecção pelo HIV <12 meses & $54,0 \%$ & $66,3 \%$ & 1,23 & $0,45-3,38$ & 0,60 \\
Internação anterior & $30,6 \%$ & $26,8 \%$ & 1,00 & $0,36-2,75$ & 0,98 \\
\hline
\end{tabular}

OR= O dds Ratio; IC 95\%= intervalo com 95\% de confiança 


\section{DISCU SSÃO}

As informações procedentes da análise dos dados de 240 pacientes com infecção pelo HIV internados em um hospital universitário, em três diferentes períodos de 12 meses (1990, 1992 e 1994) mostram que, de maneira similar à apresentação epidemiológica observada no País, trata-se essencialmente de população constituída de homens jovens, brancos, solteiros, e tendo o comportamento homossexual como principal modo de transmissão. A razão entre o sexo masculino e feminino foi de 3,6 homens para 1 mulher, a mesma observada no País nesse período ${ }^{3}$.

Chamam a atenção o aumento do período de tempo entre o diagnóstico da infecção pelo HIV e a hospitalização e o aumento do percentual de pacientes que já apresenta condições clínicas definidoras de AIDS $^{2}$ no momento da hospitalização, o que pode ser considerado como indicador de uma maior e melhor utilização da rede ambulatorial. Outro fato que fortalece essa constatação é a observação de um percentual cada vez maior de pacientes em acompanhamento nos ambulatórios especializados do hospital estudado. Por outro lado, o aumento, ao longo dos anos, do percentual de pacientes que evolui para o óbito, aponta para uma maior seletividade dos pacientes hospitalizados, internando-se pacientes em estágios mais avançados da doença e com um risco de evolução para o óbito maior nos primeiros sete dias de internação $(\mathrm{OR}=3,88)$.

Quanto ao perfil de ocorrência das infecções oportunistas e neoplasias, a candidíase oral foi a infecção oportunista definidora de $\operatorname{AIDS}^{2}$ mais freqüentemente observada, seguida da tuberculose, da pneumonia por Pneumocystis carinii e da neurotoxoplasmose. De maneira semelhante, os dados do Ministério da Sáude 3 mostram que, no momento da notificação, a candidíase $(31,4 \%)$ foi a principal infecção oportunista, seguida da pneumonia por Pneumocystis carinii $(24,8 \%)$ e toxoplasmose cerebral $(14,9 \%)$. Esses mesmos dados mostram que a tuberculose disseminada foi identificada em $14,4 \%$ dos casos enquanto a pulmonar em $11,2 \%$ (critério Rio de Janeiro / Caracas). Medidas profiláticas de eficácia comprovada, se empregadas corretamente, reduzem de forma dramática a ocorrência da pneumonia por Pneumocystis carinii, da tuberculose e da toxoplasmose cerebral ${ }^{4}$. Essas três infecções são importantes causas de internação no País, apesar de mostrarem redução nos coeficientes de incidência para os anos estudados.

Essas informações reforçam a idéia de que a disponibilidade de acompanhamento ambulatorial adequado com o emprego oportuno das medidas disponíveis de profilaxia é, certamente, o elemento mais importante na garantia da sobrevida dos pacientes com infecção pelo HIV. No presente estudo, pacientes que não fizeram acompanhamento ambulatorial na unidade tiveram três vezes mais risco de evoluir para o óbito $(\mathrm{OR}=3,29)$, sugerindo que os esforços devam ser concentrados na acessibilidade universal a serviços de qualidade, o que poderá contribuir de maneira significativa para um melhor prognóstico dos pacientes.

A partir de 1996, com a demonstração da eficácia das associações terapêuticas preconizando o uso em conjunto de inibidores da transcriptase reversa, inibidores da protease e inibidores não nucleosídeos, a atenção aos pacientes com infecção pelo HIV vem se deslocando progressivamente para a rede ambulatorial. A determinação da carga viral tem se apresentado como principal instrumento para a indicação ou ajuste terapêutico, enquanto as mono e biterapias, até então utilizadas, vêm cedendo lugar às combinações de três drogas. Esses dois avanços tecnológicos têm se mostrado decisivos na sobrevida dos pacientes com infecção pelo HIV.

Um melhor conhecimento do perfil dos pacientes com infecção pelo HIV e dos descritores clínicos e epidemiológicos da mortalidade hospitalar sobre os quais se possa intervir, podem ser úteis na tomada de decisão frente a internações para o diagnóstico e/ ou tratamento de condições clínicas conseqüentes à infecção pelo HIV, permitindo maior empenho nas intervenções a eles dirigidas. Assim, o modelo de fatores de risco proposto indica que a utilização do serviço, compreendendo não só o ingresso ao sistema de saúde, mas sobretudo de sua continuidade ${ }^{1}$, é o elemento principal na garantia da qualidade da atenção aos pacientes com infecção pelo HIV.

\section{AGRADECIMENTOS}

Ao Miguel Hijar do Ministério da Saúde e à Zulmira Hartz da Fundação Oswaldo Cruz (RJ) pelas preciosas sugestões e comentários apresentados ao texto. 


\section{REFERÊNCIAS}

1. ADAY, L.A. \& ANDERSEN, R. Marco teórico para el estudio del acceso a la atención medica. Health Serv. Res., 9:208-20, 1974

2. BOLETIM EPIDEMIOLÓGICO AIDS. Ministério da Saúde. 1992, 5(4)

3. BOLETIM EPIDEMIOLÓGICO AIDS. Ministério da Saúde. 1998, 11(1).

4. CENTERS FOR DISEASE CONTROL AND PREVENTION. Guidelines for the prevention of opportunistic infetions in persons infected with human immunodeficiency virus: a summary. $M M W R, \mathbf{4 4}(\mathrm{R}-8): 1-34,1995$.

5. DALLAL, G.E. Logistic: a logistic regression program for the IBM PC. Am. Stat.; 42:272, 1988.

6. FLEISHMAN, J.A. et al. Correlates of medical service utilization among people with HIV infection. Health Serv Res., 29:527-48, 1994.

7. GOTTLIEB, M.S. et al. Pneumocystis pneumonia: Los Angeles. Morb. Mort. Wkly Rep.,30:250-2, 1981.

8. HELLINGER, F.J. The use of health services by women with HIV infection. Health Serv. Res. 28:543-61, 1993.

9. LEMP, G.F.S. et al. Survival trends for patients with AIDS JAMA, 263:402-6, 1990.
10. MELNICK, S.L. et al. Survival and disease progression according to gender of patients with HIV infection: the Terry Beirn Community Programs for Clinical Research on AIDS. JAMA, 272:1915-21,1994.

11. MOORE, R.D. et al. Zidovudine and the natural history of the Acquired Immunodeficiency Syndrome. N. Engl. J. Med., 324:1412-6, 1991.

12. MOR, V. et al. Variation in health services use among HIVinfected patients. Med. Care, 30:17-29, 1992.

13. PIOT, P. \& MERSON, M.H. Global perspectives on HIV infection and AIDS. In: Mandell, G.L.; Bennet, J.E.; Dolin, R. Principles and practice of infectious diseases. 4 th ed. New York, Churcill Livingstone, 1995.p. 1164-74.

14. RAPPARINI, C.S. et al. Comparação clínico-epidemiológica de pacientes com SIDA internados em serviço especializado em intervalo de 6 anos. Rev. Soc. Bras. Med. Trop., 28(Supl. I):217, 1995 .

15. THULER, L.C.S. \& VAZ, L.F.G. Infecção pelo HIV. J. Bras. Med., 66:85-108, 1994.

16. TURNER, B.J. \& BALL, J.K. Variations in inpatient mortality for AIDS in a national sample of hospitals. J. Acq. Imm. Def. Syndr., 5:978-87, 1992.

17. WORLD HEALTH ORGANIZATION. Global AIDS surveillance. Weekly Epidem. Rec., 48:357-60, 1997. 